\title{
Analysis and Optimization for Weighted Sum Rate in Energy Harvesting Cooperative NOMA Systems
}

\author{
Binh Van Nguyen, Quang-Doanh Vu, and Kiseon Kim
}

\begin{abstract}
We consider a cooperative non-orthogonal multiple access system with radio frequency energy harvesting, in which a user with good channel harvests energy from its received signal and serves as a decodeand-forward relay for enhancing the performance of a user with poor channel. We here aim at maximizing the weighted sum rate of the system by optimizing the power allocation coefficient used at the source and the power splitting coefficient used at the user with good channel. By exploiting the specific structure of the considered problem, we propose a low-complexity one-dimensional search algorithm which can provide optimal solution to the problem. As a benchmark comparison, we derive analytic expressions and simple high signal-to-noise ratio (SNR) approximations of the ergodic rates achieved at the two users and their weighted sum with fixed values of the power allocation and the power splitting coefficients, from which the scaling of the weighted sum in the high SNR region is revealed. Finally, we provide representative numerical results to demonstrate the validity of our results.
\end{abstract}

Index Terms - Cooperative NOMA, RF-energy harvesting, weighted sum rate analysis and optimization.

\section{INTRODUCTION}

Non-orthogonal multiple access (NOMA) transmission is emerging as a promising multiple access technique for the next generation of wireless networks [1]. The cornerstone of NOMA is to exploit the power domain and channel quality difference among users to achieve multiple access. An issue rising in a NOMA system is that users with good channel conditions can significantly strengthen their performance, while the performance of users with

Copyright (c) 2015 IEEE. Personal use of this material is permitted. However, permission to use this material for any other purposes must be obtained from the IEEE by sending a request to pubspermissions@ieee.org.

Binh Van Nguyen is with the Institute of Research and Development, Duy Tan University, Da Nang 550000, Vietnam. He is also with the School of Electrical Engineering and Computer Science, Gwangju Institute of Science and Technology, Republic of Korea. (E-mail : binhnguyen@gist.ac.kr).

Kiseon Kim is with the School of Electrical Engineering and Computer Science, Gwangju Institute of Science and Technology, Republic of Korea. (E-mail : kskim@gist.ac.kr).

Quang-Doanh $\mathrm{Vu}$ is with the Centre for Wireless Communications, University of Oulu, Finland. (E-mail: doanh.vu@oulu.fi).

Nguyen and Kim gratefully acknowledge the support from Electronic Warfare Research Center at Gwangju Institute of Science and Technology, originally funded by Defense Acquisition Program Administration (DAPA) and Agency for Defense Development (ADD).

The work of $\mathrm{Vu}$ was supported by the projects "Flexible UplinkDownlink Resource Management for Energy and Spectral Efficiency Enhancing in Future Wireless Networks (FURMESFuN)" funded by the Academy of Finland under Grant 31089, and "6Genesis Flagship" funded by the Academy of Finland under Grant 318927. bad channel conditions are relatively poor [2]. A possible solution for this problem is combining cooperative communication with NOMA to generate a cooperative NOMA (C-NOMA) transmission scheme in which users with good channel conditions operate as relays to strengthen the transmission reliability for users suffering from bad channel conditions [3]-[6].

Recently, radio frequency energy harvesting (RF-EH) has become an efficient solution to prolong the lifetime of energy-constraint wireless communication systems [7]. The advantage of RF-EH is from the fact that RF signals carry both information and energy at the same time, i.e. RF-EH allows limited-power nodes to scavenge energy and process information simultaneously [8]. There exist two main RF-EH techniques, namely, time switching (TS) and power splitting (PS). With TS, a receiver switches between energy harvester and data decoder. With PS, a receiver separates the RF signals into two parts (one for $\mathrm{EH}$ and the other for decoding) by a PS coefficient. Here, we mainly focus on PS, since PS is considered to be more general compared to TS [9].

In C-NOMA systems, in some cases due to the limited energy at good users, i.e. in sensors and internet-of-things contexts, it may not be possible for good users to relay signals toward poor users. To alleviate this issue, RF$\mathrm{EH}$ is introduced to C-NOMA systems. Representative examples for this approach are [10]-[12]. Particularly, [10] -[11] proposed user-pair selection schemes and analyze the performance in terms of outage probability. In addition, [12] investigated the problem aiming at maximizing the achievable rate of a good user while guaranteeing the quality-of-service requirement of a poor user.

Different from [10]-[12], in this work, we focus on maximizing weighted sum rate of an RF-EH C-NOMA system which has been still relatively open. It is worth mentioning that the problem of weighted sum rate allows to prioritize users, and thus, finds many applications in wireless communications [13]-[14]. For examples, the weights can be chosen by the controller (i.e. scheduler) based on the state of the packet queues following the maxstability policy (please refer to [14] for detail discussion); or the controller determines the weights based on the throughput for the users in the previous time slots for, e.g. proportional fairness [15]. Also, the weighted sum rate problem is encountered in network utility maximization and cross-player control policies [16]. It should be noted that the results presented in [12] cannot be directly applied to our problem due to the different structures of the two problems. Specifically, our main contributions are as 
follows.

- We consider an RF-EH C-NOMA system having a source and two users. We formulate the problem of weighted sum rate maximization in which power allocation (PA) and PS coefficients are the design parameters. The problem is non-convex whose optimal solution can be found by the exhaustive twodimensional (2D) search. Towards a more efficient solution, we develop an one-dimensional (1D) search algorithm by exploiting the specific structure of the problem.

- For a comparison benchmark, we derive closed-form expressions and high signal-to-noise ratio (SNR) approximations of the ergodic rates achieved at the two users and their weighted sum with fixed PA and PS coefficients.

- We numerically demonstrate that optimized PA and PS coefficients can significantly improves the system performance in terms of weighted sum rate, i.e. $37.5 \%$ enhancement when the average SNR is $30 \mathrm{~dB}$ and the weight ratio is 5 . On the other hand, the analysis results reveal that the scaling of the weighted sum rate is $\frac{w_{1}}{2} \log _{2}(\mathrm{SNR})$, where $w_{1}$ is the priority weight of the good user.

\section{System ModeL}

We consider a wireless communication system consisting of a source, denoted by S, and two users which are associated with different channel conditions; we denote the user with good channel by $\mathrm{U}_{1}$, and the one with bad channel by $\mathrm{U}_{2}$. All nodes are equipped with a single-antenna and operate in the half-duplex mode. Let $h_{1}, h_{2}$, and $h_{3}$ denote the complex channel coefficients between $\mathrm{S}$ and $\mathrm{U}_{1}, \mathrm{~S}$ and $\mathrm{U}_{2}$, and $\mathrm{U}_{1}$ and $\mathrm{U}_{2}$, respectively. All channels are assumed to be independent and identically distributed Rayleigh block fading. From the assumption about channel quality, we have $g_{1} \geq g_{2}$ where $g_{i}=\left|h_{i}\right|^{2}$.

We focus on the transmission from $\mathrm{S}$ to the users. The transmission protocol includes two phases, each of length $T$ in time unit. In particular, let $x_{i}, i \in\{1,2\}$, be the normalized complex signal for $\mathrm{U}_{i}$, and $P_{\mathrm{S}}$ be the transmit power at $\mathrm{S}$. In the first phase, $\mathrm{S}$ generates a superimposed signal given by $x_{\mathrm{S}}=\sqrt{\alpha P_{\mathrm{S}}} x_{1}+\sqrt{(1-\alpha) P_{\mathrm{S}}} x_{2}$, where $\alpha$ denotes the PA coefficient, and broadcasts $x_{\mathrm{S}}$ to the users. The received signal at $\mathrm{U}_{i}$ during this phase is

$$
y_{i}=h_{i} x_{\mathrm{S}}+n_{i}
$$

where $n_{i}$ is the additive white Gaussian noise (AWGN) with variance $N_{0}$ at the note $\mathrm{U}_{i}$.

User $\mathrm{U}_{1}$ uses its received signal for decoding $x_{1}$, harvesting energy, and decoding $x_{2}$. In particular, $\mathrm{U}_{1}$ divides $y_{1}$ into two parts with a PS coefficient $\rho \in[0,1]$. The first part given by $y_{1}^{\mathrm{eh}}=\sqrt{\rho} y_{1}$ is for harvesting energy, and the second part given by $y_{1}^{\mathrm{ip}}=\sqrt{1-\rho} y_{1}$ is for decoding information. Consequently, the energy harvested at $U_{1}$ is [11]

$$
E_{1}=T \eta \rho P_{\mathrm{S}} g_{1}
$$

where $\eta$ denotes the energy conversion efficiency. $\mathrm{U}_{1}$ decodes $x_{2}$ based on $y_{1}^{\text {ip }}$, then applies successive interference cancellation (SIC) before decoding $x_{1}$. Therefore, the signal-to-interference-plus-noise ratios (SINRs) for decoding $x_{2}$ and $x_{1}$ at $\mathrm{U}_{1}$ are as follows

$$
\begin{aligned}
& \gamma_{1}^{x_{2}}(\alpha, \rho)=\frac{(1-\rho)(1-\alpha) P_{\mathrm{S}} g_{1}}{(1-\rho) \alpha P_{\mathrm{S}} g_{1}+(1-\rho) N_{0}+\mu N_{0}}, \\
& \gamma_{1}^{x_{1}}(\alpha, \rho)=\frac{(1-\rho) \alpha P_{\mathrm{S}} g_{1}}{(1-\rho) N_{0}+\mu N_{0}},
\end{aligned}
$$

respectively. Here, the last term in the denominator of $\gamma_{1}^{x_{2}}(\alpha, \rho)$ and $\gamma_{1}^{x_{1}}(\alpha, \rho)$ are due to the conversion noise which is assumed to be AWGN with variance $\mu N_{0}$ [17].

In the second phase, $\mathrm{U}_{1}$ uses the harvested energy $E_{1}$ to transmit $x_{2}$ to $\mathrm{U}_{2}$. The signal received at $\mathrm{U}_{2}$ during this phase is

$$
\tilde{y}_{2}=\sqrt{\rho \eta P_{\mathrm{S}} g_{1}} h_{3} x_{2}+n_{2},
$$

where we, following recent related works, have assumed that the harvested energy is used for information forwarding only, while the energy for maintaining circuit and signal processing is neglected [10]-[12]. We suppose that the maximal ratio combining (MRC) receiver is used at $\mathrm{U}_{2}$ [18]. Then the SINR for decoding $x_{2}$ at $\mathrm{U}_{2}$ is

$$
\gamma_{2}^{\mathrm{MRC}}(\alpha, \rho)=\frac{(1-\alpha) P_{\mathrm{S}} g_{2}}{\alpha P_{\mathrm{S}} g_{2}+N_{0}+\mu N_{0}}+\frac{\rho \eta P_{\mathrm{S}} g_{1} g_{3}}{N_{0}+\mu N_{0}} .
$$

In summary, the instantaneous achieved rates at $\mathrm{U}_{1}$ and $\mathrm{U}_{2}$ are $C_{1}(\alpha, \rho)=\frac{1}{2} \log _{2}\left(1+\gamma_{1}^{x_{1}}(\alpha, \rho)\right)$ and $C_{2}(\alpha, \rho)=$ $\frac{1}{2} \log _{2}\left(1+\min \left\{\gamma_{1}^{x_{2}}(\alpha, \rho), \gamma_{2}^{\mathrm{MRC}}(\alpha, \rho)\right\}\right)$, respectively.

\section{Weighted Sum Rate Optimization}

Our aim is to maximize the weighted sum rate of the system. Particularly, the optimization problem is formulated as

$$
\begin{aligned}
& \underset{\alpha, \rho}{\operatorname{maximize}} w_{1} C_{1}(\alpha, \rho)+w_{2} C_{2}(\alpha, \rho) \\
& \text { subject to } 0<\alpha<1,0 \leq \rho \leq 1,
\end{aligned}
$$

where $w_{1}>0$ and $w_{2}>0$ are the priority weights. ${ }^{1}$ Here we focus on the case $w_{2}>w_{1}$ since the optimal solution for the case $w_{2} \leq w_{1}$ is trivial, i.e. it is not difficult to justify that the optimal solution for this case is $(\alpha=1, \rho=0)$. A practical example for the considered scenario is that in cellular network, the user at cell-edge suffering bad channel conditions for a long time will be assigned a larger weight compared to the one in near base station area for fairness and/or stability [13]-[14].

Objective function (7a) is non-convex with respect to the related variables. For achieving an optimal solution, an exhaustive 2D search procedure (over $\alpha$ and $\rho$ ) can be used. Clearly, doing this is highly complex and inefficient. In the following, by looking inside the problem, we develop a low-complexity 1D search algorithm which solves (7) optimally.

\footnotetext{
${ }^{1}$ The weights are the given parameters which is determined by the controllers for a specific policy, e.g. buffer stability or proportional fairness.
} 
We start with an useful result stated as follows.

Lemma 1. Let $\left(\alpha^{*}, \rho^{*}\right)$ be an optimal of $(7)$, then

$$
C_{2}\left(\alpha^{*}, \rho^{*}\right)=\frac{1}{2} \log _{2}\left(1+\gamma_{2}^{\mathrm{MRC}}\left(\alpha^{*}, \rho^{*}\right)\right) .
$$

Proof: The lemma can be proved by contradiction, i.e. we first assume that there is an optimal point such that (8) does not hold then we show that such the point does not exist. More specifically, suppose that there exists an optimal point $\left(\alpha^{*}, \rho^{*}\right)$ such that

$$
\log _{2}\left(1+\gamma_{1}^{x_{2}}\left(\alpha^{*}, \rho^{*}\right)\right)<\log _{2}\left(1+\gamma_{2}^{\mathrm{MRC}}\left(\alpha^{*}, \rho^{*}\right)\right) .
$$

Clearly, it must be $\rho^{*}>0$ due to the assumption $g_{1} \geq g_{2}$. Now, we observe that $\gamma_{2}^{\mathrm{MRC}}(\alpha, \rho)$ and $\gamma_{1}^{x_{2}}(\alpha, \rho)$ are increasing and decreasing functions of $\rho$, respectively. And $\gamma_{1}^{x_{2}}(\alpha, 0)>\gamma_{2}^{\mathrm{MRC}}(\alpha, 0)$. Consequently, we always can find $\Delta \rho>0$ such that $\rho^{*}-\Delta \rho \geq$ 0 , and $\gamma_{1}^{x_{2}}\left(\alpha^{*}, \rho^{*}-\triangle \rho\right)=\gamma_{2}^{\mathrm{MRC}}\left(\alpha^{*}, \rho^{*}-\triangle \rho\right)$. Since $\gamma_{1}^{x_{2}}\left(\alpha^{*}, \rho^{*}-\triangle \rho\right)>\gamma_{1}^{x_{2}}\left(\alpha^{*}, \rho^{*}\right)$, we have $C_{2}\left(\alpha^{*}, \rho^{*}-\right.$ $\triangle \rho)>C_{2}\left(\alpha^{*}, \rho^{*}\right)$. Moreover, $C_{1}\left(\alpha^{*}, \rho^{*}-\triangle \rho\right)>C_{1}\left(\alpha^{*}, \rho^{*}\right)$ because $\gamma_{1}^{x_{1}}(\alpha, \rho)$ is a decreasing function of $\rho$. Consequently, we have $w_{1} C_{1}\left(\alpha^{*}, \rho^{*}-\triangle \rho\right)+w_{2} C_{2}\left(\alpha^{*}, \rho^{*}-\right.$ $\triangle \rho)>w_{1} C_{1}\left(\alpha^{*}, \rho^{*}\right)+w_{2} C_{2}\left(\alpha^{*}, \rho^{*}\right)$. This means the point $\left(\alpha^{*}, \rho^{*}-\triangle \rho\right)$ achieves a better objective value compared to $\left(\alpha^{*}, \rho^{*}\right)$, which contradicts the assumption at the beginning of the proof that $\left(\alpha^{*}, \rho^{*}\right)$ is an optimal. This implies that, at the optimal, we always have $\log _{2}\left(1+\gamma_{1}^{x_{2}}\left(\alpha^{*}, \rho^{*}\right)\right) \geq \log _{2}\left(1+\gamma_{2}^{\mathrm{MRC}}\left(\alpha^{*}, \rho^{*}\right)\right)$. This completes the proof.

From Lemma 1 and the monotonicity of the logarithmic function, we can rewrite (7) as

$$
\begin{array}{cl}
\underset{\alpha, \rho}{\operatorname{maximize}} & f(\alpha, \rho) \\
\text { subject to } & \gamma_{1}^{x_{2}}(\alpha, \rho) \geq \gamma_{2}^{\mathrm{MRC}}(\alpha, \rho) \\
& 0<\alpha<1,0 \leq \rho<1
\end{array}
$$

where $f(\alpha, \rho) \triangleq\left(1+\gamma_{1}^{x_{1}}(\alpha, \rho)\right)\left(1+\gamma_{2}^{\mathrm{MRC}}(\alpha, \rho)\right)^{\tilde{w}_{2}}$, and $\tilde{w}_{2}=w_{2} / w_{1}$. As a further step, we equivalently rewrite (10) as

$$
\begin{aligned}
& \underset{\alpha, \rho}{\operatorname{maximize}} f(\alpha, \rho) \\
& \text { subject to } 0<\alpha<1,0 \leq \rho \leq \tilde{\rho}(\alpha),
\end{aligned}
$$

where $\tilde{\rho}(\alpha)=\frac{b-\sqrt{b^{2}-4 a c}}{2 a}, \bar{\gamma}=P_{\mathrm{S}} / N_{0}, a=\frac{\eta \bar{\gamma} g_{1} g_{3}\left(\alpha \bar{\gamma} g_{1}+1\right)}{1+\mu}$, $b=\frac{\eta \bar{\gamma} g_{1} g_{3}\left(\alpha \bar{\gamma} g_{1}+\mu+1\right)}{1+\mu}-\frac{(1-\alpha) \bar{\gamma} g_{2}\left(\alpha \bar{\gamma} g_{1}+1\right)}{\alpha \bar{\gamma} g_{2}+\mu+1}+(1-\alpha) \bar{\gamma} g_{1}$, and $c=(1-\alpha) \bar{\gamma} g_{1}-\frac{(1-\alpha) \bar{\gamma} g_{2}\left(\alpha \bar{\gamma} g_{1}+1+\mu\right)}{\alpha \bar{\gamma} g_{2}+\mu+1}$. The equivalence can be proved as follows. We first note that the left handside (LHS) of (10b) monotonically increases while the right hand-side (RHS) of (10b) monotonically decreases with $\rho$. In addition, when $\rho=0$, the RHS is larger than the LHS due to the assumption $g_{1} \geq g_{2}$. Moreover, the RHS $\rightarrow 0$ when $\rho \rightarrow 1$. Thus, given $\alpha \in(0,1)$, there exists an unique $\tilde{\rho}(\alpha) \in(0,1)$ such that $(10 \mathrm{~b})$ is satisfied if and only if $\rho \in[0, \tilde{\rho}(\alpha))$. It is noting that $(10 \mathrm{~b})$ can be written as $a \rho^{2}-b \rho+c \geq 0$, from which we yield $\tilde{\rho}(\alpha)$. The new bound $\tilde{\rho}(\alpha)$ in $(11 \mathrm{~b})$ plays the important role in developing the proposed algorithm.

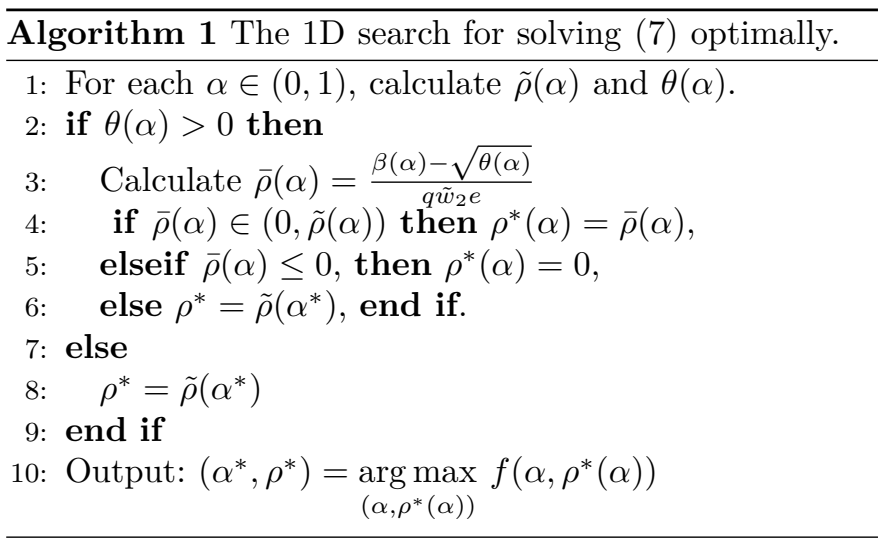

We now focus on objective function (11a). For a given $\alpha$, (11a) reduces to a function of $\rho$ given as

$$
f_{\alpha}(\rho) \triangleq \frac{(d-e \rho)}{t-\rho}(p+q \rho)^{\tilde{w}_{2}}
$$

where $d=1+\mu+\alpha \bar{\gamma} g_{1}, e=1+\alpha \bar{\gamma} g_{1}, t=1+\mu, p=$ $1+\frac{(1-\alpha) \bar{\gamma} g_{2}}{\alpha \bar{\gamma} g_{2}+1+\mu}, q=\frac{\eta \bar{\gamma} g_{1} g_{3}}{1+\mu}$. We also introduce a function of $\alpha$ given as

$$
\theta(\alpha)=\beta^{2}-q \tilde{w}_{2} e\left(d p-e p t+q \tilde{w}_{2} t d\right)
$$

where $\beta(\alpha)=0.5 q d\left(\tilde{w}_{2}-1\right)+0.5 q e t\left(\tilde{w}_{2}+1\right)$. We have an useful property of $f_{\alpha}(\rho)$ stated as follows.

Proposition 1. If $\theta(\alpha)>0$ and $\bar{\rho}(\alpha)=\frac{\beta-\sqrt{\theta(\alpha)}}{q \tilde{w}_{2} N_{0} v} \in(0,1)$, when $\rho$ increases, $f_{\alpha}(\rho)$ increases until reaches a maximum at $\bar{\rho}(\alpha)$ then decreases. If $\theta(\alpha)>0$ and $\bar{\rho}(\alpha) \leq 0, f_{\alpha}(\rho)$ is decreasing over $\rho \in(0,1)$. Otherwise, $f_{\alpha}(\rho)$ is increasing over $\rho \in(0,1)$.

The proof of the proposition can be easily obtained via the gradient of $f_{\alpha}(\rho)$ given as

$$
\frac{\partial f_{\alpha}(\rho)}{\partial \rho}=\frac{\left[(d-e t)(p+q \rho)+q \tilde{w}_{2}(t-\rho)(d-e \rho)\right]}{(p+q \rho)^{1-\tilde{w}_{2}}(t-\rho)^{2}} .
$$

The algebraic steps are skipped for the sake of brevity.

The property allows us to find the optimal value of $\rho$ when the optimal value $\alpha^{*}$ is given as follows. $\rho^{*}=\bar{\rho}\left(\alpha^{*}\right)$ if $\theta\left(\alpha^{*}\right)>0$ and $0<\bar{\rho}\left(\alpha^{*}\right)<\tilde{\rho}\left(\alpha^{*}\right)$. If $\theta\left(\alpha^{*}\right)>0$ and $\bar{\rho}\left(\alpha^{*}\right)<0, \rho^{*}=0$. Otherwise $\rho^{*}=\tilde{\rho}\left(\alpha^{*}\right)$. In summary, we outline the proposed 1D search procedure in Algorithm 1 which outputs the optimal solution of (7).

\section{ERgodic Rate Analysis}

In this section, we derive the ergodic rates achieved at the users (and their weighted sum) with fixed values of $\alpha$ and $\rho$, which can be used as a benchmark in evaluating the Algorithm 1.

Before going into detail, it is important to note that the cumulative distributed function (CDF) of the ordered variables $g_{1}$ and $g_{2}$ are given by $F_{g_{1}}(x)=F_{\tilde{g}_{1}}(x) F_{\tilde{g}_{2}}(x)$ and $F_{g_{2}}(x)=1-\left[1-F_{\tilde{g}_{1}}(x)\right]\left[1-F_{\tilde{g}_{2}}(x)\right]$, where $F_{\tilde{g}_{1}}(x)$ and $F_{\tilde{g}_{2}}(x)$ are the CDF of unordered variables $\tilde{g}_{1}$ and $\tilde{g}_{2}$. 


\section{A. Ergodic Rate of $\mathrm{U}_{1}$}

The ergodic rate of the $\mathrm{U}_{1}$ is expressed as follows [18]

$$
C_{1}^{\mathrm{e}}=\frac{1}{2 \ln (2)} \int_{0}^{\infty} \frac{1-F_{X}(x)}{1+x} d x,
$$

where $X=\frac{(1-\rho) \alpha \bar{\gamma} g_{1}}{1-\rho+\mu}$, and $F_{X}(x)$ is given by

$$
F_{X}(x)=1-\sum_{i=1,2} \exp \left(-\frac{A x}{\delta_{i}^{2}}\right)+\exp \left(-\frac{A x}{\delta_{12}^{2}}\right),
$$

where $\delta_{i}^{2}=d_{i}^{-\epsilon_{i}}$ is the power of the unordered channel $\tilde{h}_{i}$, $d_{i}$ and $\epsilon_{i}$ denote the distance and the pathloss exponent, $\delta_{12}^{2}=\delta_{1}^{2} \delta_{2}^{2} /\left(\delta_{1}^{2}+\delta_{2}^{2}\right)$, and $A=(1-\rho+\mu) /(1-\rho) \alpha \bar{\gamma}$. Plugging (16) into (15) gives

$$
C_{1}^{e}=\frac{1}{2 \ln (2)} \sum_{i=1,2,12} \psi(i) \exp \left(\frac{A}{\delta_{i}^{2}}\right) \Gamma\left(0, \frac{A}{\delta_{i}^{2}}\right),
$$

where $\psi(1)=1, \psi(2)=1, \psi(12)=-1$, and $\Gamma(x, y)$ is the incomplete upper Gamma function.

\section{B. Ergodic Rate of $\mathrm{U}_{2}$}

Similar to (15), we have

$$
C_{2}^{\mathrm{e}}=\frac{1}{2 \ln (2)} \int_{0}^{\infty} \frac{1-F_{Z}(z)}{1+z} d z,
$$

where $Z=\min \left\{\gamma_{1}^{x_{2}}(\alpha, \rho), \gamma_{2}^{\mathrm{MRC}}(\alpha, \rho)\right\}=\min \{Y, W\}$ and $F_{Z}(z)$ can be approximated as

$$
F_{Z}(z) \simeq 1-\operatorname{Pr}[Y>z] \operatorname{Pr}[W>z],
$$

where the correlation between $Y$ and $W$ is ignored. It can be readily verified that the correlation between $Y$ and $W$ vanishes in the high SNR region implying that the approximation is tight when the average SNR goes large. The probability term $\operatorname{Pr}[Y>z]$ is first derived as

$$
\operatorname{Pr}[Y>z]=\left\{\begin{array}{l}
0, \quad \text { if } z \geq \frac{1-\alpha}{\alpha}, \\
\sum_{i=1,2,12} \psi(i) \exp \left(-\frac{\alpha A z}{\delta_{i}^{2}(1-\alpha-\alpha z)}\right), \text { if } z<\frac{1-\alpha}{\alpha} .
\end{array}\right.
$$

Secondly, $\operatorname{Pr}[W>z]$ can be approximated as follows

$$
\operatorname{Pr}[W>z] \simeq 1-\int_{0}^{z} F_{W_{1}}(z-y) f_{W_{2}}(y) d y,
$$

where $W_{1}=\frac{(1-\alpha) \bar{\gamma} g_{2}}{\alpha \bar{\gamma} g_{2}+1+\mu}, W_{2}=\frac{\rho \eta \bar{\gamma} g_{1} g_{3}}{1+\mu}$, and

$$
\begin{aligned}
& F_{W_{1}}(z)=\left\{\begin{array}{l}
1, \text { if } z \geq \frac{1-\alpha}{\alpha}, \\
1-\exp \left(-\frac{z(1+\mu)}{\bar{\gamma} \delta_{12}^{2}(1-\alpha-\alpha z)}\right), \text { if } z<\frac{1-\alpha}{\alpha},
\end{array}\right. \\
& f_{W_{2}}(z)=2 \sum_{i=1,2,12} \psi(i) \frac{B}{\delta_{i}^{2}} K_{0}\left(2 \sqrt{\frac{B z}{\delta_{i}^{2}}}\right),
\end{aligned}
$$

where $B=(1+\mu) / \rho \eta \bar{\gamma} \delta_{3}^{2}$ and $K_{n}(x)$ denotes the modified Bessel function of the second kind of order $n^{\text {th }}$ [19]. (20) is obtained by ignoring the correlation between $W_{1}$ and $W_{2}$ which is vanished in the high SNR region. We note that $(z-y)$ is always less than $\frac{1-\alpha}{\alpha}$ when $z<\frac{1-\alpha}{\alpha}$. On the other hand, when $z \geq \frac{1-\alpha}{\alpha}, z-y \geq \frac{1-\alpha}{\alpha}$ if $y \leq z-\frac{1-\alpha}{\alpha}$ and $z-y<\frac{1-\alpha}{\alpha}$ if $z-\frac{1-\alpha}{\alpha} \leq y \leq z$. Base on this fact, we can further extend (21) as follows

$$
\begin{aligned}
& \operatorname{Pr}[W>z] \simeq 1-F_{W_{2}}(z) \\
& +\int_{L(z)}^{z} \exp \left(-\frac{(1+\mu)(z-y)}{\bar{\gamma} \delta_{12}^{2}(1-\alpha-\alpha z+\alpha y)}\right) f_{W_{2}}(y) d y,
\end{aligned}
$$

where $L(z)=0$ if $z<(1-\alpha) / \alpha, L(z)=z-(1-\alpha) / \alpha$ otherwise, and

$$
F_{W_{2}}(z)=1-2 \sum_{i=1,2,12} \psi(i) \sqrt{\frac{B z}{\delta_{i}^{2}}} K_{1}\left(2 \sqrt{\frac{B z}{\delta_{i}^{2}}}\right) .
$$

Plugging (24) and (20) into (19) and (18), we obtain

$$
\begin{aligned}
C_{2}^{e} \simeq & \int_{0}^{\frac{1-\alpha}{\alpha}} \frac{1 / \ln (2)}{1+z} \sum_{i=1,2,12} \psi(i) \sqrt{\frac{B z}{\delta_{i}^{2}}} K_{1}\left(2 \sqrt{\frac{B z}{\delta_{i}^{2}}}\right) \\
& \cdot \sum_{i=1,2,12} \psi(i) \exp \left(\frac{-\alpha A z}{\delta_{i}^{2}(1-\alpha-\alpha z)}\right) d z \\
& +\int_{0}^{\frac{1-\alpha}{\alpha}} \int_{L(z)}^{z} \frac{1 / \ln (2)}{1+z} \exp \left(\frac{-(1+\mu)(z-y)}{\bar{\gamma} \delta_{12}^{2}(1-\alpha-\alpha z+\alpha y)}\right) \\
& \cdot \sum_{i=1,2,12} \psi(i) \exp \left(\frac{-\alpha A z}{\delta_{i}^{2}(1-\alpha-\alpha z)}\right) \\
& \cdot \sum_{i=1,2,12} \psi(i) \frac{B}{\delta_{i}^{2}} K_{0}\left(2 \sqrt{\frac{B y}{\delta_{i}^{2}}}\right) d y d z,
\end{aligned}
$$

which can be readily evaluated by using Matlab or Mathematica. From (17) and (26), we can straightforwardly obtain the system weighted sum rate, i.e. $C_{\text {sum }}^{\mathrm{e}}=w_{1} C_{1}^{\mathrm{e}}+$ $w_{2} C_{2}^{\mathrm{e}}$, with fixed values of $\alpha$ and $\rho$.

\section{High SNR Analysis}

To gain novel insights from our afore-presented analytic results, we now investigate the ergodic rates in the high SNR region.

Proposition 2. In the high SNR region, the ergodic rates of $\mathrm{U}_{1}$ and $\mathrm{U}_{2}$ can be approximated as follows

$$
\begin{aligned}
C_{1}^{\mathrm{e}} & \approx \frac{1}{2 \ln (2)}\left[\ln \left(\frac{\delta_{1}^{2}+\delta_{2}^{2}}{A}\right)-\chi\right], \\
C_{2}^{\mathrm{e}} & \approx \frac{1}{2} \log _{2}\left(1+\frac{1-\alpha}{\alpha}\right),
\end{aligned}
$$

where $\chi$ denote the Euler constant.

Proof: For $C_{1}^{\mathrm{e}}$, we first note that $\Gamma(0, x)=-E i(-x)$, where $\operatorname{Ei}(x)$ denotes the exponential integral function. Then using the the facts that $\exp (x) \stackrel{x \rightarrow 0}{\longrightarrow} 1$ and $E i(x) \stackrel{x \rightarrow 0}{\longrightarrow} \chi+\ln (-x)+x$, we can obtain (27). For $C_{2}^{\mathrm{e}}$, let's first recall its instantaneous expression $C_{2}=\frac{1}{2} \log _{2}\left(1+\min \left\{\gamma_{1}^{x_{2}}(\alpha, \rho), \gamma_{2}^{\mathrm{MRC}}(\alpha, \rho)\right\}\right)$. Then, in the high region of $\bar{\gamma}$, we can readily show that $\gamma_{1}^{x_{2}}(\alpha, \rho) \rightarrow$ $\frac{1-\alpha}{\alpha}<\gamma_{2}^{\operatorname{MRC}}(\alpha, \rho) \rightarrow \frac{1-\alpha}{\alpha}+\frac{\rho \eta \bar{\gamma} g_{1} g_{3}}{1+\mu}$, from which (28) can be obtained. 


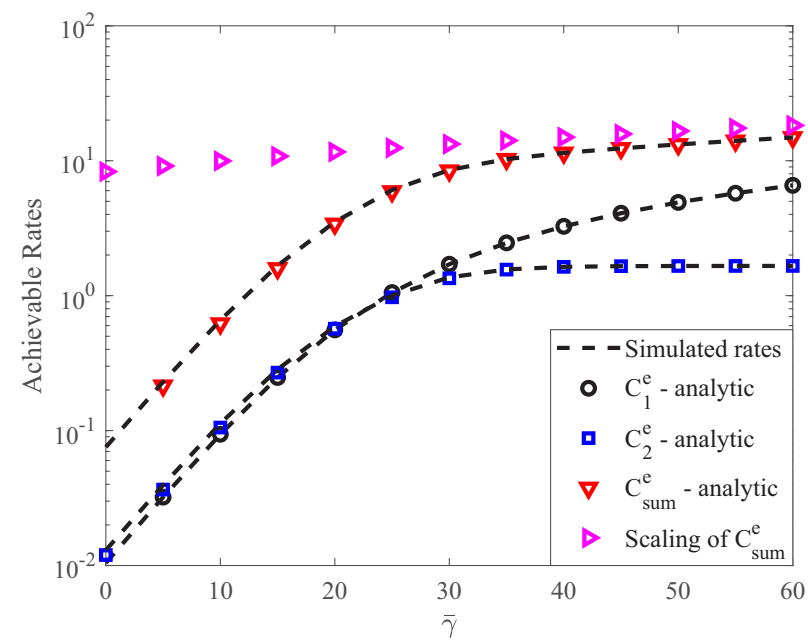

Figure 1. Achievable rates with fixed values of $\alpha$ and $\rho$.

Proposition 2 implies that as the average SNR $\bar{\gamma}$ increases, the ergodic rate of $\mathrm{U}_{1}$ monotonically increases, however, that of $\mathrm{U}_{2}$ is saturated. This is reasonable because as $\bar{\gamma}$ increases, the SNR used for decoding $x_{1}$ at $U_{1}$ also increases, and thus, the ergodic rate of $U_{1}$ increases. On the other hand, the actual SINR used for decoding $x_{2}$ is limited by the minimum of the SINRs used for decoding $x_{2}$ at $\mathrm{U}_{1}$ and $\mathrm{U}_{2}$. In addition, when $\bar{\gamma}$ increases, the SINR used for decoding $x_{2}$ at $\mathrm{U}_{1}$ quickly converges to $\frac{1-\alpha}{\alpha}$ and limits the actual SINR used for decoding $x_{2}$, which makes the ergodic rate of $\mathrm{U}_{2}$ saturated.

From Proposition 2, we have

$$
C_{\mathrm{sum}}^{\mathrm{e}}=w_{1} C_{1}^{\mathrm{e}}+w_{2} C_{2}^{\mathrm{e}} \approx \frac{w_{1}}{2} \log _{2}(\bar{\gamma}),
$$

which reveals that when $\bar{\gamma} \rightarrow \infty$, the scaling of the system weighted sum rate is $\frac{w_{1}}{2} \log _{2}(\bar{\gamma})$. In other words, (29) shows that the weighted sum rate increases log-linearly with the increase of the average SNR $\bar{\gamma}$.

\section{Numerical Results and Discussions}

We now provide representative simulated results to validate our analysis and demonstrate the enhancement of the system performance achieved by the proposed 1D algorithm. In our simulations, we set $\eta=0.7, \epsilon_{i}=3$, $T=1$, and the coordinates of source, good user, and poor user are $(0,0),(1,1)$, and $(3,0)$, respectively.

Figure 1 plots the ergodic rates of the considered system with fixed values of $\alpha$ and $\rho$. The first observation is that the analytic curve of $C_{1}^{\mathrm{e}}$ follows the corresponding simulated one excellently, while the analytic curves of $C_{2}^{\mathrm{e}}$ and $C_{\text {sum }}^{\mathrm{e}}$ quickly converge to the corresponding simulated curves in the medium and high SNR regions. This result implies that our analyses on the system's ergodic rates are valid. Secondly, the figure confirms our finding on the scaling of the weighted sum rate in the high SNR region. The other interesting observation is that the ergodic rate of $\mathrm{U}_{2}$ is saturated as the average SNR gets large, revealing that increasing the average SNR (or equally increasing the

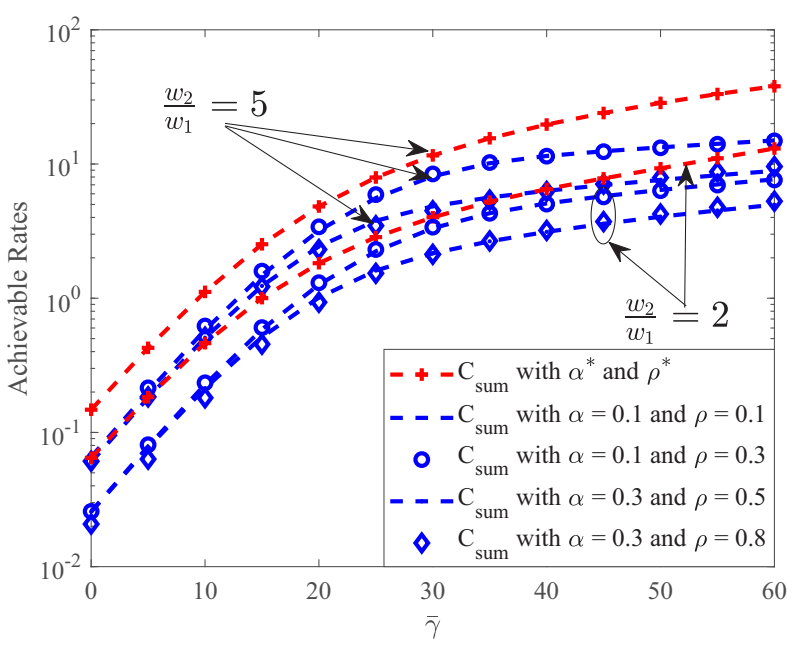

Figure 2. Weighted sum rate with optimal and fixed values of $\alpha$ and $\rho$.

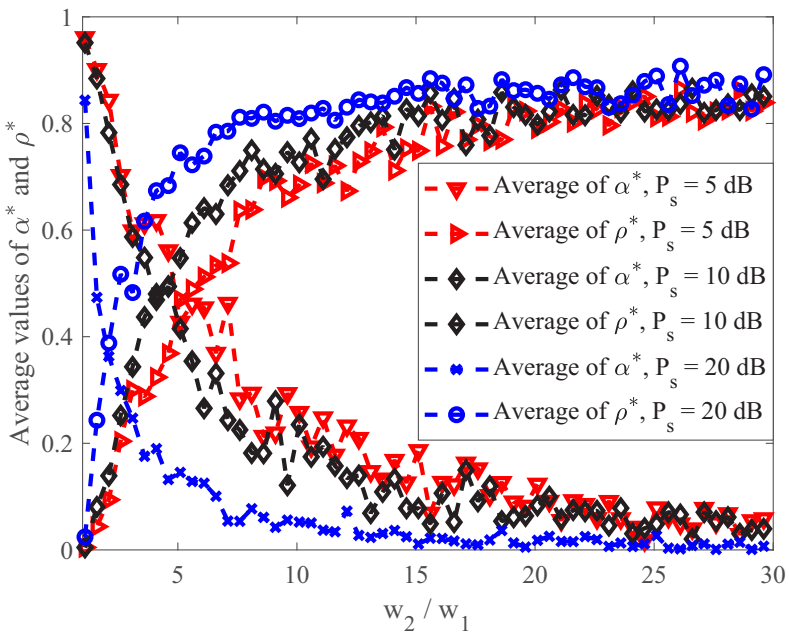

Figure 3. Average value of $\rho^{*}$ and $\alpha^{*}$ versus $\tilde{w}_{2}$.

transmit power $P_{\mathrm{S}}$ ) cannot enhance the performance of the user with poor channel.

Figure 2 plots the system weighted sum rates with optimal and fixed values of $\alpha$ and $\rho$ as functions of the average SNR. We take $\tilde{w}_{2}=\{2,5\}$. The figure clearly shows that using the Algorithm 1 remarkably enhances the weighted sum rate of the system. Particularly, at $\bar{\gamma}=30$ $\mathrm{dB}$, optimal values of $\alpha$ and $\rho$ provides $37.5 \%$ and $18.4 \%$ weighted sum rate enhancements with $\tilde{w}_{2}=5$ and $\tilde{w}_{2}=2$. Thus, the results strongly suggest that the parameters $\alpha$ and $\rho$ should be optimized.

In Fig. 3, we illustrate the average of the optimal values of $\alpha$ and $\rho$ (i.e. $\mathbb{E}\left\{a^{*}\right\}$ and $\mathbb{E}\left\{\rho^{*}\right\}$, respectively) versus $\tilde{w}_{2}$. An interesting observation is that as $\tilde{w}_{2}$ increases, $\mathbb{E}\left\{a^{*}\right\}$ reduces and approaches zero. This is due to the fact that when $\tilde{w}_{2}$ enlarges, $\mathrm{U}_{2}$ has a higher priority compared to $\mathrm{U}_{1}$, and thus, more power should be allocated to the transmission of $x_{2}$. On the other hand, we can also observe that $\mathbb{E}\left\{\rho^{*}\right\}$ increases and tends to a certain value. This is because the rate of $U_{2}$ provided in Lemma 1 is an increasing function with $\rho$, and $\rho^{*}$ should be small enough 
so that the constraint $(10 \mathrm{~b})$ is satisfied.

\section{Conclusion}

We considered a C-NOMA system with RF-EH including a source and two users. We first developed a 1D search algorithm to optimally solve the problem of weighted sum rate maximization respect to the power allocation $\alpha$ and the power splitting coefficient $\rho$. Then, we derived closedform expressions and high SNR approximations of the ergodic rates achieved at the two users with fixed values of $\alpha$ and $\rho$. The numerical results demonstrated that using the optimal values of $\alpha$ and $\rho$ significantly enlarges the system weighted sum rate, i.e. $37.5 \%$ enhancement when the average SNR is $30 \mathrm{~dB}$ and the weight ratio is 5 . In addition, we revealed that the scaling of the weighted sum rate with fixed value of $\alpha$ and $\rho$ is $\frac{w_{1}}{2} \log _{2}(\bar{\gamma})$ in the high SNR region.

\section{REFERENCES}

[1] L. Zhang, J. Liu, M. Xiao, G. Wu, Y. Liang, and S. Li, "Performance Analysis and Optimization in Downlink NOMA Systems with Cooperative Full-Duplex Relaying," IEEE J. Select. Areas Commun., vol. 35, no. 10, pp. 2398-2412, Oct. 2017.

[2] Z. Yang, Z. Ding, Y. Wu, and P. Fan, "Novel Relay Selection Strategies for Cooperative NOMA," IEEE Trans. Veh. Technol., vol. 66, no. 11, pp. 10114-10123, Nov. 2017.

[3] Z. Ding, M. Peng, and H. V. Poor, "Cooperative Non-orthogonal Multiple Access in 5G Systems," IEEE Commun. Lett., vol. 19 , no. 8, pp. 1462-1465, Aug. 2015.

[4] J. Kim and I Lee, "Capacity Analysis of Cooperative Relaying Systems using Non-orthogonal Multiple Access," IEEE Commun. Lett., vol. 19, no. 11, pp. 1949-1952, Nov. 2015.

[5] D. Wan, M. Wen, H. Yu, Y. Liu, F. Ji, and F. Chen, "Nonorthogonal Multiple Access for Dual-Hop Decode-and-Forward Relaying," in Proc. IEEE Global Communication Conference, Washington, USA, Dec. 2016.

[6] J. Men, J. Ge, and C. Zhang, "Performance Analysis of Non-orthogonal Multiple Access For Relaying Networks over Nakagami-m Fading Channels," IEEE Trans. Veh. Technol., vol. 66, no. 2, pp. 1200-1208, Apr. 2016.

[7] X. Fafoutis, A. D. Mauro, C. Orfanidis, and N. Dragoni, "Energy-Efficient Medium Access Control for Energy Harvesting Communications," IEEE Trans. Consum. Electron., vol. 61, no. 4, pp. 402-410, Nov. 2015.

[8] F. Wang, S.Guo, Y. Yang, and B. Xiao, "Relay Selection and Power Allocation for Cooperative Network With Energy Harvesting," IEEE Sys. J., vol. pp, no. 99, pp. 1-12, April 2016.

[9] Q. Shi, L. Liu, W. Xu and R. Zhang, "Joint Transmit Beamforming and Receive Power Splitting for MISO SWIPT Systems," IEEE Trans. Wireless Commun., vol. 13, no. 6, pp. 3269-3280, June 2014.

[10] Y. Liu, Z. Ding, M. Elkashlan, and H. V. Poor, "Cooperative Non-orthogonal Multiple Access with Simultaneous Wireless Information and Power Transfer," IEEE J. Sel. Areas Commun., vol. 34, no. 4, pp. 938-953, April 2016.

[11] N. T. Do, D. B. da Costa, T. Q. Duong, and B. An, "A BNBF User Selection Scheme for NOMA-based Cooperative Relaying Systems with SWIPT," IEEE Commun. Lett., vol. 21, no. 3, pp. 664-667, Mar. 2017.

[12] Y. Xu, C. Shen, Z. Ding, X. Sun, S. Yan, G. Zhu, and Z. Zhong, "Joint Beamforming and Power-Splitting Control in Downlink Cooperative SWIPT NOMA Systems," IEEE Trans. Sig. Process., vol. 65, no. 18, pp. 4874-4886, Sep. 2017.

[13] S. S. Christensen, R. Agarwal, E. D. Carvalho and J. M. Cioffi, "Weighted sum-rate maximization using weighted MMSE for MIMO-BC beamforming design," IEEE Trans. Wireless Commun., vol. 7 , no. 12 , pp. 4792-4799, Dec. 2008.

[14] M. Kobayashi and G. Caire, "An Iterative Water-Filling Algorithm for Maximum Weighted Sum-Rate of Gaussian MIMOBC," IEEE Sel. Areas Commun., vol. 24, no. 8, pp. 1640-1646, Aug. 2006
[15] L. Tran, M. Bengtsson and B. Ottersten, "Iterative Precoder Design and User Scheduling for Block-Diagonalized Systems," IEEE Trans. Sig. Process., vol. 60, no. 7, pp. 3726-3739, July 2012.

[16] Pradeep Chathuranga Weeraddana, Marian Codreanu, Matti Latva-aho, Anthony Ephremides and Carlo Fischione, "Weighted Sum-Rate Maximization in Wireless Networks: A Review" Foundations and Trends in Networking, vol. 6, no. 1-2, pp. 1-163, 2012.

[17] B. V. Nguyen, H. Jung, D. Har, and K. Kim, "Performance Analysis of a Cognitive Radio Network With an Energy Harvesting Secondary Transmitter Under Nakagami- $m$ Fading," IEEE Access, vol. 6, pp. 4135-4144, Jan. 2018.

[18] X. Yue, Y. Liu, S. Kang, A. Nallanathan, Z. Ding, "Exploiting Full/Half-Duplex User Relaying in NOMA Systems, "IEEE Trans. Commun., vol. 66, no. 2, pp. 560-575, Sep. 2017.

[19] I. S. Gradshteyn and I. M. Ryzhik, "Table of Integrals, Series, and Products $7^{\text {th }}$ Edition," Acadamic Press, 2007. 